\title{
Static one-leg standing balance test as a screening tool for low muscle mass in healthy elderly women
}

\author{
Praval Khanal ${ }^{1,2}\left(\mathbb{0} \cdot\right.$ Lingxiao He $^{1,2} \cdot$ Georgina K. Stebbings $^{1} \cdot$ Gladys L. Onambele-Pearson $^{1} \cdot$ Hans Degens $s^{3,4}$. \\ Alun G. Williams ${ }^{1,5} \cdot$ Martine Thomis $^{2} \cdot$ Christopher I. Morse $^{1}$
}

Received: 11 November 2020 / Accepted: 17 February 2021 / Published online: 13 March 2021

(c) The Author(s) 2021

\begin{abstract}
Background Identification of simple screening tools for detecting lower skeletal muscle mass may be beneficial for planning effective interventions in the elderly.

Aims We aimed to (1) establish a threshold for one-leg standing balance test (OLST) time for low muscle mass, and (2) test the ability of that threshold to assess muscular impairments in a poor balance group.

Methods Eyes-open OLST (maximum duration $30 \mathrm{~s}$ ) was performed with right and left legs in 291 women (age $71 \pm 6$ years). OLST time was calculated as the sum of the OLST time of right and left legs. Fat-free mass (FFM), skeletal muscle mass (SMM), fat mass, biceps brachii and vastus lateralis sizes; handgrip strength (HGS), elbow flexion maximum torque $\left(\mathrm{MVC}_{\mathrm{EF}}\right)$ and knee extension maximum torque $\left(\mathrm{MVC}_{\mathrm{KE}}\right)$ were measured. Muscle quality was calculated as $\mathrm{MVC}_{\mathrm{KE}} / \mathrm{FFM}$ and physical activity was assessed by questionnaire. Low muscle mass was defined as $\mathrm{SMM}_{\text {relative }}$ of $22.1 \%$, a previously established threshold for pre-sarcopenia.

Results The OLST threshold time to detect low muscle mass was $55 \mathrm{~s}$ (sensitivity: 0.63 ; specificity: 0.60 ). The poor balance group (OLST $<55 \mathrm{~s}$ ) had higher fat mass $(3.0 \%, p<0.001)$, larger VL thickness $(5.1 \%, p=0.016)$, and lower HGS $(-10.2 \%$, $p<0.001), \mathrm{MVC}_{\mathrm{EF}}(-8.2 \%, p=0.003), \mathrm{MVC}_{\mathrm{KE}}(-9.5 \%, p=0.012), \mathrm{MVC}_{\mathrm{KE}} / \mathrm{FFM}(-11.0 \%, p=0.004)$ and physical activity $(-8.0 \%, p=0.024)$ compared to the normal balance group. While after adjusting age, the differences exist for HGS, fat mass and VL thickness only.

Discussion An OLST threshold of $55 \mathrm{~s}$ calculated as the summed score from both legs discriminated pre-sarcopenic characteristics among active, community-dwelling older women with limited potential (sensitivity 0.63 , specificity 0.60 ).

Conclusion OLST, which can be performed easily in community settings without the need for more complex muscle mass measurement, may help identify women at risk of developing sarcopenia.
\end{abstract}

Keywords Pre-sarcopenia $\cdot$ One-leg standing balance $\cdot$ Screening tool $\cdot$ Skeletal muscle phenotypes

Praval Khanal

praval.khanal@gmail.com

1 Department of Sport and Exercise Sciences, Manchester Metropolitan University, Manchester M15 6BH, UK

2 Department of Movement Sciences, Physical Activity, Sports and Health Research Group, KU Leuven, Leuven, Belgium

3 Division of Health Science, Manchester Metropolitan University, Manchester, UK

4 Institute of Sport Science and Innovations, Lithuanian Sports University, Kaunas, Lithuania

5 Institute of Sport, Exercise and Health, University College London, London, UK

\section{Introduction}

Sarcopenia is characterized by loss of skeletal muscle mass and muscle strength that contributes to a decline in physical performance with ageing [1]. The presence of sarcopenia is dependent on the elderly falling below thresholds of muscle strength and mass to levels rarely seen in those living independently. Skeletal muscle-related impairments such as functional impairment and physical disability may result due to low skeletal muscle mass [2] before reaching these sarcopenic thresholds. Therefore, early identification of skeletal muscle mass below a pre-sarcopenic threshold (i.e., $22.1 \%$ skeletal muscle mass relative to body mass, hereafter termed "low muscle mass" [2]), may facilitate appropriate 
interventions-primarily related to combination of nutrition and exercise [3]-to reverse or slow down the process of muscle wasting and prolong independent living. A simple screening tool that could be conducted with minimal equipment could help to identify those elderly individuals with low muscle mass, who are at risk of sarcopenia and subsequent frailty and loss of independence. One would expect interventions to be more effective at pre-sarcopenic levels of muscle mass than at the accepted thresholds for developed sarcopenia as by then limitations in a physical function may prevent meaningful improvements to the above functional impairment thresholds. Current sarcopenia thresholds for muscle mass are derived with techniques such as magnetic resonance imaging (MRI), bioimpedance analysis (BIA) or dual-energy X-ray absorptiometry (DXA) [1] which may not be universally available. At the most basic functional level, even assessing handgrip strength (a valid measure of the functional aspect of sarcopenia) requires a specialist equipment [4]. An alternative and more accessible screening assessment for low muscle mass in the elderly, that could be adopted in populations living independently is the static one-leg standing balance test (OLST).

Postural stability, an important parameter to explain the state of neuromuscular health, describes an individual's ability to maintain postural control during a stable position, voluntary movement and reaction to external disturbances without falling [5]. The reduction in dynamic and static balance with ageing is multifaceted, and attributed to both the central nervous and neuromuscular system [6] with fewer receptor cells in vestibular organs [7], alteration in sensory perception and slowing of reaction times [8]. Ultimately, it is the inability of the neuromuscular system to respond to minor postural perturbations that likely result in the association between weak muscles and poor balance with ageing. While several testing procedures for the assessment of balance impairment with ageing exist, the static OLST is easy to adopt in clinical and geriatric settings to assess postural steadiness in a static position [9]. Although there is inconsistency in the use of single leg or summed times, maximum duration of tests, and eye condition (opened or closed) among existing studies [10], the OLST has been successfully associated with muscle strength and frailty $[11,12]$. To date, the OLST has been adopted as a screening tool for balance impairment [10], whereby failing to pass a $5 \mathrm{~s}$ threshold has been associated with poor functional status [13] and impaired ability to perform activities of daily living (ADLs) [14]. Importantly, it should be noted that this $5 \mathrm{~s}$ threshold has been derived mostly in either very weak and frail populations or older populations [13-15]. Falls and fall-related injuries are the primary cause of hospitalization among the elderly and signal the initial stage of loss of independence [16] and show an association with both lower skeletal muscle mass [17] and poor balance [18]. Therefore, identification of an OLST-time threshold for pre-sarcopenic risk in active community dwellers may allow an alternative screening method to evaluate musculoskeletal health and via subsequent interventions reduce the risk of adverse outcomes such as injurious falls associated with low muscle mass.

While the $5 \mathrm{~s}$ threshold is associated with poor functional status in weak and frail populations, this threshold is unable to detect low muscle mass because active, independently living older people will exceed this threshold easily [19]. Furthermore, since there can be significant differences in OLST time between left and right legs [20], a summed score of both legs in a balance test maybe more appropriate for identifying the threshold risk of low muscle mass. Thus, we reasoned that for the detection of low muscle mass a different OLST threshold is needed that could be recommended in clinical and geriatric settings if associated with other clinically relevant pre-sarcopenic characteristics in older individuals with poor balance. Therefore, the aim of the present study was to (1) identify an OLST time threshold for pre-sarcopenic risk of low muscle mass, (2) test how the identified OLST time threshold is associated with the pre-sarcopenic characteristics body composition, regional muscle size, muscle strength, muscle quality and physical activity level.

\section{Materials and methods}

\section{Participant characteristics and inclusion criteria}

Older women ( $n=291,60-91$ years, age $71 \pm 6$ years) were mainly recruited via the University of the Third Age, Cheshire region, UK, or via word-of-mouth. All participants were $60+$ years, self-reported as being free from any issues that affected their daily activities and physical independence. Individuals were excluded if they had a history of neuromuscular or cardiovascular conditions, including a self-reported history of vertigo.

\section{One-leg standing balance test}

The one-leg standing balance test (OLST) was performed with both the right and left leg alternately. Participants were asked to stand unshod and then to stand on one leg, whilst flexing the contralateral knee at $90^{\circ}$ behind them, whilst maintaining a vertical thigh position, parallel to the standing leg. The test was performed alternately with the right and left leg with $10 \mathrm{~s}$ rest between trials. If they completed $30 \mathrm{~s}$ of one-leg standing (recorded with stopwatch), then it was recorded as OLST for that specific leg [21]. If they did not achieve $30 \mathrm{~s}$, they were allowed a maximum of three attempts until they achieved $30 \mathrm{~s}$, or when they failed to achieve $30 \mathrm{~s}$ the maximum time among 
the three attempts was recorded as their specific leg OLST. OLST total was calculated as the sum of OLST time of the right OLST (RL) and left leg OLST (LL): OLST $=$ OLST $(\mathrm{RL})+$ OLST (LL). Test-re-test reliability is moderate $(\mathrm{ICC}=0.60)$ [22] to high $(\mathrm{ICC}>0.90)$ [23] for the OLST.

\section{Body composition and muscle size}

Skeletal muscle mass (SMM) and fat mass were estimated by bio-impedance analysis (Model 1500; Bodystat, Isle of Man, UK) as described in our previous work [24].

During the procedure, participants lay in a supine position for 5 min on a physiotherapist bed to ensure homogeneous distribution of body fluid, followed by attaching the adhesive electrodes to the dorsum of the right hand and leg. Subsequently, a small current was passed between the attached electrodes.

Skeletal muscle mass was estimated using an equation previously validated in a Caucasian population [25] as:

Skeletal muscle mass $(\mathrm{SMM})=\mathrm{Ht}^{2} /(R \quad \times$ 0.401) $-0.071 \times$ age +5.102 ,

Where, $\mathrm{Ht}$ is height of the individual in $\mathrm{m}, R$ is resistance from the device in Ohm and age is in years. The SMM has shown a high correlation with DXA measures [26]. Subsequently, fat-free mass (FFM) was calculated from the recorded fat mass as body mass-fat mass.

An ultrasound $\left(\mathrm{MyLab}^{\mathrm{TM}}\right.$ Twice, Esaote Biomedical, Italy) was used to perform the scan to measure the biceps brachii thickness at $60 \%$ length from the proximal end of the humerus $[27,28]$.

For vastus lateralis (VL) thickness, the origin and insertion of the VL were identified and sagittal scans at 50\% $\mathrm{VL}$ length were performed. The mean of three thicknesses measured at proximal, middle and distal points between superficial and deep aponeuroses was recorded as VL thickness.

\section{Muscle strength and quality}

Hand grip strength (HGS) was measured with a dynamometer (JAMAR plus, JLW Instruments, Chicago, USA) as described in our previous work [27].

Similarly, maximum isometric elbow flexion torque $\left(\mathrm{MVC}_{\mathrm{EF}}\right)$ and knee extension torque $\left(\mathrm{MVC}_{\mathrm{KE}}\right)$ were measured with dynamometer with a load cell (Zemic, EtenLeur, Netherlands). The detailed procedure has been described in our previous work [27].

Lower limb muscle quality was defined as the knee strength relative to fat-free mass $\left(\mathrm{MVC}_{\mathrm{KE}} / \mathrm{FFM}\right)$ [29].

\section{Physical activity}

The PASE (Physical activity scale for elderly) questionnaire was used to evaluate physical activity level [30].

\section{Assessment of low muscle mass}

Low muscle mass was defined as having a relative skeletal muscle mass $\left(\mathrm{SMM}_{\mathrm{r}}\right)<22.1 \%$ [2], calculated as $100 \times \mathrm{SMM} /$ Body mass. Based on this threshold for low muscle mass, the corresponding OLST threshold was calculated (see below).

\section{Statistical analysis}

Statistical analyses were carried out using SPSS Version 26.0 for Windows (IBM Corp., Armonk, NY, USA) and $p<0.05$ was considered statistically significant. Throughout, low muscle mass was defined as individuals with $\mathrm{SMM}_{\mathrm{r}}<22.1 \%$ [2]. Kruskal-Wallis tests were performed for continuous variables for the group-wise comparisons with Bonferroni correction to adjust for multiple comparisons. Spearman correlations were performed to identify the strength of association of OLST with \%SMM, age and BMI; and BMI with muscle-related phenotypes. Variables significantly correlated were used in multivariable linear regression analysis to predict the variance of the OLST. To establish the threshold value of OLST that predicted presarcopenic risk, receiver operating characteristic (ROC) curve analysis was performed. OLST time threshold was defined as that with the highest values of sensitivity and specificity for distinguishing between low muscle mass and healthy muscle mass participants. The area under the ROC curve was used to assess the discriminatory ability of the model. Based on the ROC analysis, participants were then divided into those of "poor" (summed OLST $<55 \mathrm{~s}$, see below) and "normal" (summed OLST $\geq 55 \mathrm{~s}$ ) balance. Kolmogorov-Smirnov tests were completed to check for normal distribution of neuromuscular phenotypes in the poor balance and normal balance groups. For phenotypes meeting parametric assumptions (with both poor balance and normal balance groups), between-group analyses were conducted using independent samples $t$ tests. For phenotypes that violated parametric assumptions, Mann-Whitney tests were used and Monte-Carlo $p$ values are reported. Further to these, analysis of covariance (ANCOVA) was also used to investigate if the differences remained after using age as covariate. Unless stated otherwise, parametric data are presented as mean $\pm S D$, with non-parametric data presented as median (inter-quartile range). 


\section{Results}

\section{Distribution of participants with low muscle mass prevalence and balance performance}

Fifty-five percent ( $n=161)$ of the participants passed the 30 s OLST with both right and left legs, with older groups showing a lower $60 \mathrm{~s}$ pass prevalence than younger groups (Table 1). OLST time was lower in older age groups, such that the $80+$ years group only achieved $29 \%$ of OLST time achieved by the $60-64$ years group (Fig. 1). Only one participant was identified as sarcopenic (having both lower skeletal muscle mass $\left(\% \mathrm{SMM}_{\mathrm{r}}<22.1 \%\right)$ and lower HGS (HGS $<19 \mathrm{~kg}$ )) in the current older population. The prevalence of $\% \mathrm{SMM}_{\mathrm{r}}$ low muscle mass increased (though unevenly) with age from $8 \%$ in the 60-64 year group to $29 \%$ in the $80+$ years group (Table 1 ). Most participants $(n=261,90 \%)$ exceeded 5 s OLST on both legs.

Table 1 Distribution of participants across age groups and their categorizations according to body mass index, \% skeletal muscle mass, achieving maximum $60 \mathrm{~s}$ one-leg standing balance test and low muscle mass prevalence

\begin{tabular}{llllll}
\hline Age-group (years) & $\begin{array}{l}\text { Number of par- } \\
\text { ticipants }\end{array}$ & BMI $\left(\mathrm{kg} / \mathrm{m}^{2}\right)$ & \% $\mathrm{SMM}_{\mathrm{r}}$ & $\begin{array}{l}\text { \% individuals failing to achieve } \\
\text { summed 60 s OLST }\end{array}$ & $\begin{array}{l}\text { Low muscle mass preva- } \\
\text { lence using } \mathrm{SMM}_{\mathrm{r}} \\
{[\%(n)]}\end{array}$ \\
\hline $60-64$ & 40 & $25.2 \pm 4.0$ & $27.2 \pm 3.7$ & $10.0 \%$ & $7.5 \%(3)$ \\
$65-69$ & 104 & $26.3 \pm 4.9$ & $25.7 \pm 3.7$ & $36.5 \%$ & $16.3 \%(17)$ \\
$70-74$ & 96 & $25.8 \pm 3.9$ & $25.4 \pm 4.3$ & $51.0 \%$ & $13.5 \%(13)$ \\
$75-79$ & 34 & $25.1 \pm 3.3$ & $26.0 \pm 3.3$ & $67.6 \%$ & $5.9 \%(2)$ \\
$80+$ & 17 & $28.4 \pm 3.4$ & $23.6 \pm 3.1$ & $94.1 \%$ & $29.4 \%(5)$ \\
All participants & $n=291$ & $25.9 \pm 4.2$ & $25.8 \pm 3.9$ & $45.0 \%$ & $13.7 \%(40)$ \\
\hline
\end{tabular}

$B M I$ body mass index, OLST one-leg standing balance test $S M M$ skeletal muscle mass. Data are presented as mean $\pm \mathrm{SD}$ for $\mathrm{BMI}$ and $\% \mathrm{SMM}$

Fig. 1 Boxplot showing OLST time for five 5-year age groups. Circles and asterisks indicate outliers and extreme outliers, respectively. $O L S T$ one-leg standing balance test. $1,2,3,4,5$ show difference from 60-64 years, $65-69$ years, $70-74$ years, $75-79$ years and $80+$ years, respectively (Kruskal-Wallis test with Bonferroni correction for multiple comparisons) at $p<0.05$

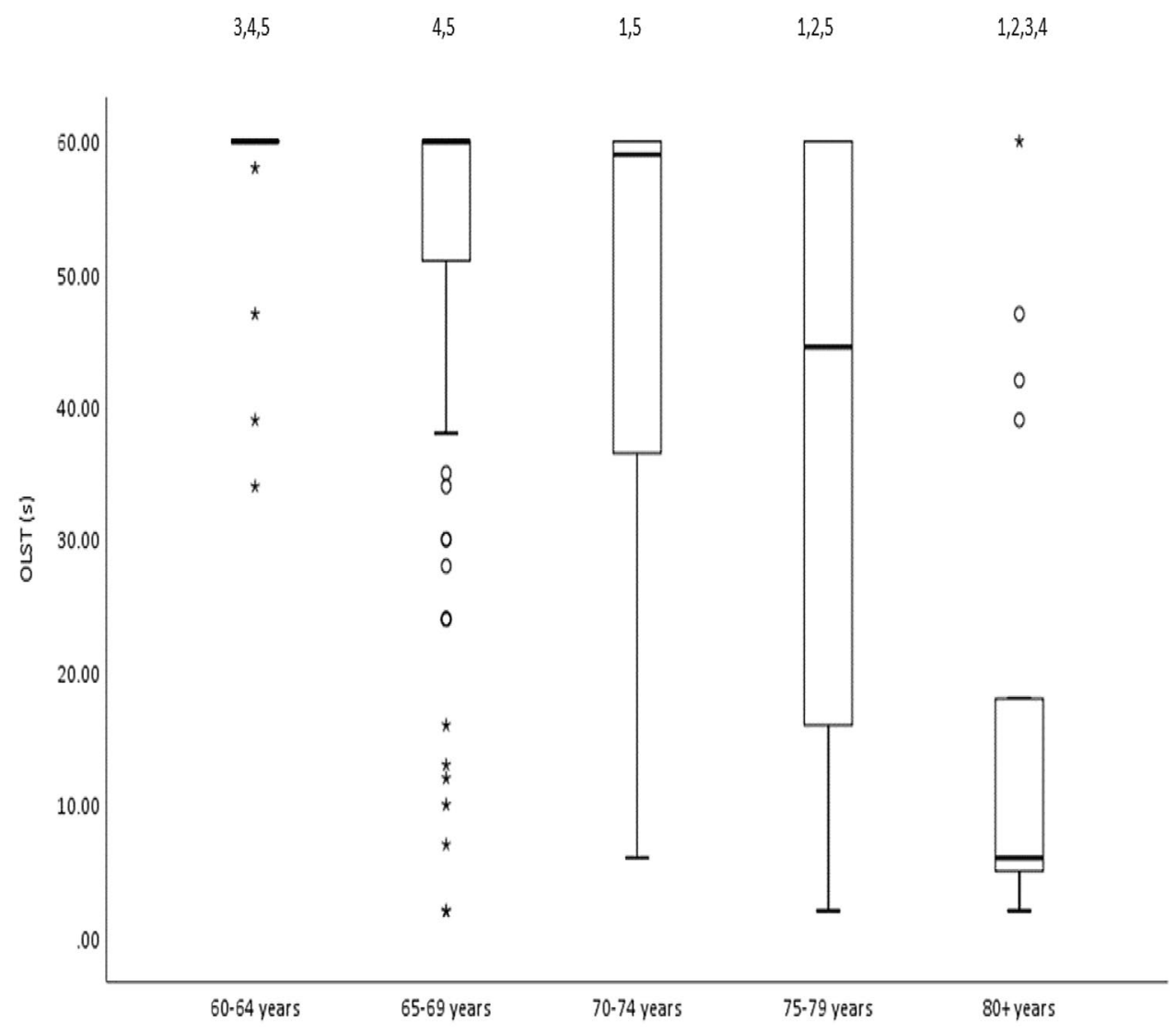




\section{Association of \% SMMr, OLST, body composition and muscle related phenotypes}

OLST time was moderately and positively correlated with $\%$ $\operatorname{SMM}_{\mathrm{r}}(\rho=0.352, p<0.001)$ and moderately and negatively with BMI $(\rho=-0.320, p<0.001)$ and age $(\rho=-0.446$, $p<0.001)$. Similarly, BMI was moderately and positively correlated with biceps brachii thickness $(\rho=0.226$, $p<0.001)$ and VL-thickness $(\rho=0.311, p<0.001)$, strongly and positively correlated with fat mass $(\rho=917, p<0.001)$, and moderately and negatively correlated with $\mathrm{MVC}_{\mathrm{KE}} /$ FFM $(\rho=-0.236, p<0.001)$ while it was not correlated with HGS $(\rho=0.037, p=0.526), \operatorname{MVC}_{\mathrm{EF}}(\rho=0.111$, $p=0.058), \operatorname{MVC}_{\mathrm{KE}}(\rho=0.011, p=0.853)$ and physical activity $(\rho=-0.079, p=0.181)$. Multivariable linear regression with BMI and $\% \mathrm{SMM}_{\mathrm{r}}$ included in the model as predictors predicted approximately $14 \%$ of the variance in OLST time.

Upon ROC analysis that predicted the low muscle mass threshold of $22.1 \% \mathrm{SMM}_{\mathrm{r}}$, the AUC for the predicted model was 0.65 (Fig. 2). The threshold for OLST time was $55 \mathrm{~s}$ that corresponded to the highest sensitivity and specificity values (sensitivity 0.63 ; specificity 0.60 ).

\section{The OLST threshold and low muscle mass features}

The prevalence of poor balance (below $55 \mathrm{~s}$ OLST) was $40 \%$ $(n=117)$. Compared to the normal balance group, the poor

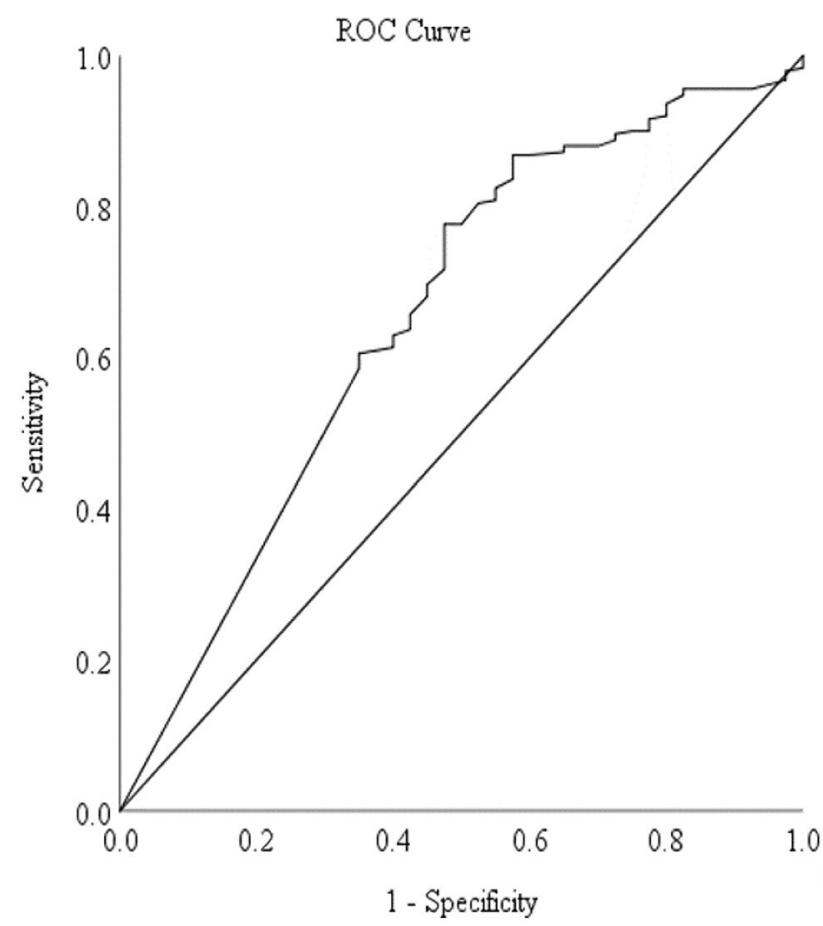

Fig. 2 ROC analysis for OLST time threshold based on low muscle mass threshold balance group showed lower HGS $(-10.2 \%, p<0.001)$, $\mathrm{MVC}_{\mathrm{EF}}(-8.3 \%, p=0.008), \mathrm{MVC}_{\mathrm{KE}}(-9.5 \%, p=0.012)$, $\mathrm{MVC}_{\mathrm{KE}} / \mathrm{FFM}(-11.0 \%, p=0.004)$ and physical activity level $(-8.0 \%, p=0.024)$ (Table 2). Similarly, there was a higher fat mass $(3.0 \%, p<0.001)$ and larger VL thickness $(5.1 \%, p=0.016)$ in the poor balance group, but no differences in biceps brachii thickness $(p=0.325)$ (Table 2). The poor balance group was older and heavier than the normal balance group (Table 2, $p<0.05$ ).

With age used as covariate, the differences remain between the poor and normal balance groups for HGS ( $F$ $(1,288)=11.3, p=0.001)$, VL-thickness $(F(1,288)=13.8$, $p<0.001)$ and fat mass $(F(1,288)=35.4, p<0.001)$ but not with $\operatorname{MVC}_{\mathrm{EF}}(F(1,288)=2.4, p=0.121), \operatorname{MVC}_{\mathrm{KE}}(F$ $(1,287)=0.4, p=0.512)$, physical activity level $(F(1$, $287)=3.4, p=0.067)$ and $\mathrm{MVC}_{\mathrm{KE}} / \mathrm{FFM}(F(1,288)=2.245$, $p=0.135)$.

\section{Discussion}

The current study identified an OLST time threshold for low muscle mass risk in healthy elderly women and then tested the potential of this derived OLST threshold to distinguish pre-sarcopenic characteristics in the poor balance group. We identified a low muscle mass OLST threshold of $55 \mathrm{~s}$ $(\mathrm{OLST}=$ OLST $(\mathrm{RL})+$ OLST $(\mathrm{LL}) ; 30$ s maximum duration for each leg) in healthy community-dwelling women. The derived 55 s OLST time threshold successfully defined a poor balance group with greater fat mass and lower muscle strength (HGS). We, therefore, suggest that the static one-leg standing balance test could be an accessible alternative to detect presence of low muscle mass in community-dwelling healthy elderly women.

Low-muscle mass is the first stage of sarcopenia detection [1] and is linked to adverse outcome measures such as physical dependence [2] and increased risk of falls [17]. However, the assessment of skeletal muscle mass requires equipment such as MRI, BIA and DXA, which may not be readily available and certainly lack the flexibility to screen for the presence of low muscle mass within a community setting in independently living elderly women. Several alternatives have previously been suggested for the detection of low muscle mass and sarcopenia. For instance, threshold measures of muscle thickness (sensitivity 0.74 ; specificity 0.17 ) [31], hand grip strength (sensitivity 0.61 ; specificity $0.60)$ [32] and anthropometric indicators such as calf circumference (sensitivity 0.6 ; specificity 1.0 ) [31] have been proposed as thresholds in sarcopenia identification. Indeed, HGS is the initial screening method for sarcopenia in elderly women based on EWGSOP2 guidelines [4], but requires a handgrip dynamometer. In contrast, OLST as in the present study requires no more than a timer and can be performed 
Table 2 Participant characteristics of poor and normal balance groups based on the identified threshold of $55 \mathrm{~s}$

\begin{tabular}{llll}
\hline Phenotypes & General characteristics & \multicolumn{2}{l}{ Standing balance test categories } \\
\cline { 4 - 4 } & $(n=291)$ & Poor balance $(n=117)$ & $\begin{array}{l}\text { Normal } \\
\text { balance } \\
(n=174)\end{array}$ \\
\hline Age (years) & & $68.8(5.7)$ \\
Body mass $(\mathrm{kg})$ & $70.6(5.7)$ & $72.7(7.7) * *$ & $63.6(10.9)$ \\
Height $(\mathrm{m})$ & $65.5(13.9)$ & $67.8(14.2) * *$ & $1.60 \pm 0.05$ \\
BMI $\left(\mathrm{kg} / \mathrm{m}^{2}\right)$ & $1.60 \pm 0.06$ & $1.59 \pm 0.06$ & $24.6(4.5)$ \\
FFM $(\mathrm{kg})$ & $25.3(4.6)$ & $26.4(6.2) * *$ & $37.7 \pm 4.9$ \\
Fat $(\mathrm{kg})$ & $37.8 \pm 5.0$ & $38.0 \pm 5.2$ & $25.7(7.8)$ \\
Fat $\%$ & $27.1(8.8)$ & $29.1(10.6) * *$ & $40.9 \pm 4.6$ \\
SMM $(\mathrm{kg})$ & $42.5 \pm 5.1$ & $44.9 \pm 4.9 *$ & $17.0 \pm 2.4$ \\
$\% \mathrm{SMM}$ & $16.6 \pm 2.2$ & $26.8 \pm 3.9$ \\
Biceps brachii thickness $(\mathrm{cm})$ & $1.72(0.44)$ & $24.3 \pm 3.3 *$ & $1.73(0.42)$ \\
VL thickness $(\mathrm{cm})$ & $1.91 \pm 0.35$ & $1.69(0.48)$ & $1.87 \pm 0.34$ \\
$\mathrm{HGS}(\mathrm{kg})$ & $30.1 \pm 4.9$ & $1.97 \pm 0.36 *$ & $31.3 \pm 4.7$ \\
MVC $_{\mathrm{EF}}(\mathrm{N} \cdot \mathrm{m})$ & $24.5(8.0)$ & $28.1 \pm 4.7 * *$ & $25.0(8.0)$ \\
MVC $_{\mathrm{KE}}(\mathrm{N} \cdot \mathrm{m})$ & $55.7 \pm 18.5$ & $23.2(7.5) *$ & $57.9 \pm 18.5$ \\
Physical activity $_{\mathrm{MVC}_{\mathrm{KE}} / \mathrm{FFM}(\mathrm{N} \cdot \mathrm{m} / \mathrm{kg})}$ & $157 \pm 50$ & $52.4 \pm 18.1 *$ & $160(60)$ \\
\hline B & $1.48 \pm 0.48$ & $141(65) *$ & $1.55 \pm 0.48$ \\
\hline
\end{tabular}

$B M I$ Body mass index, FFM fat-free mass, $S M M$ skeletal muscle mass, $V L$ vastus lateralis, $H G S$ handgrip strength, $M V C_{E F}$ isometric elbow flexion, $M V C_{K E}$ isometric knee extension. Parametric data are presented as mean $\pm \mathrm{SD}$ and non-parametric as median (inter-quartile range). ${ }^{*}$ and $* *$ denote $p<0.05$ and $p<0.001$, respectively easily and reliably by the participant and investigator [10]. Although the risk of falls during the test should not be ignored, a $5 \mathrm{~s}$ threshold in even the frailest elderly is a testament to the safety of the approach.

The $22.1 \% \mathrm{SMM}_{\mathrm{r}}$ threshold for the presence of low muscle mass used in the present study has been previously used to investigate genetic variants associated with sarcopenia [24], and identifying risk of disability [2]. In the present study, OLST was moderately and negatively associated with both age and BMI, while moderately and positively with $\% \mathrm{SMM}_{\mathrm{r} .}$ The decrement in OLST time with ageing has been reported before [21] and the positive association of $\% \mathrm{SMM}_{\mathrm{r}}$ with OLST time observed in the present study is in line with other studies showing a better physical performance with higher muscle mass [33, 34]. In the present study, establishing a low muscle mass threshold for OLST was based on ROC analysis using the $22.1 \% \mathrm{SMM}_{\mathrm{r}}$ threshold. We observed an acceptable model prediction $(\mathrm{AUC}=0.65)$ [35] between summed OLST and the $22.1 \% \mathrm{SMM}_{\mathrm{r}}$ presarcopenic threshold, and identified 55 s OLST time as a threshold with slightly lower sensitivity (0.63) and specificity (0.60). The lower AUC of the model and lower specificity and sensitivity of the OLST time threshold identified may be explained by the confounding impact of BMI on OLST time $(\rho=-0.320, p<0.001)$ and the positive correlation between $\% \mathrm{SMM}_{\mathrm{r}}$ and OLST time $(\rho=0.352, p<0.001)$. In addition, we should note that $\% \mathrm{SMM}_{\mathrm{r}}$ and BMI combined, predicted
$14 \%$ of the variance observed for OLST. The negative relation between BMI and OLST observation corresponds with a previous association of higher BMI with lower postural control and balance ability [36]. Thus, BMI may act as confounding factor affecting the physiological parameters of the balance and control in addition to reducing $\% \mathrm{SMM}_{\mathrm{r}}$ (as increment in BMI increases the body mass in denominator while calculating $\% \mathrm{SMM}_{\mathrm{r}}$ ). Previous studies have shown a positive association of physical performance measures with muscle mass and negative associations with obesity-related indices [37, 38]. One should realise, however, that there is most likely a threshold muscle mass below which OLST may also become limited by the low muscle mass. An upper limit is essential for maintaining the practicality of the test, so future studies could investigate alternatives. However, we should also note that the observed low AUC, sensitivity and specificity may be attributed to other factors related to the nervous system, alteration in sensory perception and slowness in reaction times that are associated with ageing [6-8].The 55 s OLST threshold of low muscle mass in the present study should be considered alongside existing OLST thresholds. A $5 \mathrm{~s}$ OLST threshold is established in frail and clinical populations $[10,13,15]$ and $9 \mathrm{~s}$ for locomotive dysfunction in a Japanese population [39]. In all these instances the lower OLST thresholds are from frail $(5 \mathrm{~s},[10,13,15])$ and older participants ( $9 \mathrm{~s}$ [39]). For instance, the present study included younger participants $(71 \pm 6$ vs $77 \pm 6$ years 
[39]) scored from both legs [summed score (OLST (RL) and OLST (LL)] time vs one leg OLST time) and included independently living participants, in contrast to those with locomotive dysfunction previously [39]. The derived $55 \mathrm{~s}$ OLST is therefore, more applicable for healthy older women who do not have other comorbidities and would ordinarily slip under the radar for dedicated interventions for sarcopenia prevention.

The secondary aim of the current study was to evaluate if the derived $55 \mathrm{~s}$ OLST threshold is a suitable pre-screening tool for muscle function, muscle mass and physical activity that have known implications for independence in the elderly. Participants with an OLST below the $55 \mathrm{~s}$ threshold had a higher fat mass, larger VL and lower muscle strength (HGS) than their normal-balance counterparts. The lower HGS and higher fat mass observed in the poor balance group has been previously associated with lower skeletal muscle mass [40, 41], and poor scores in functional, psychological and social health domains [42]. The larger VL muscle thickness in the poor balance group in the present study is consistent with greater muscle mass in overweight individuals [43] (high BMI observed in poor balance group in the present elderly women). Furthermore, note that muscle thickness is not the same as muscle quantity and ignores intramuscular fat deposition in obese individuals [44]. The ability to characterize pre-sarcopenic features in our poor balance group suggests the $55 \mathrm{~s}$ OLST threshold has potential to screen for pre-sarcopenia in healthy community-dwelling women.

Although the $55 \mathrm{~s}$ OLST threshold was somewhat successful in characterizing pre-sarcopenic characteristics, the authors suggest its practical use should be cautious considering the observed sensitivity, specificity and AUC values. The sensitivity and specificity may improve if the test was lengthened to $60 \mathrm{~s}$ for each leg (summed $120 \mathrm{~s}$ ), as 55\% participants completed the summed $60 \mathrm{~s}$. We also suggest that thresholds could be population-specific and could be different if alternative indices for low muscle mass are used. We also like to acknowledge that our sample represents a likely healthy bias of participants, and a volunteer bias for those active elderly as all such investigations into health and activity of elderly. Although we adopted an inclusive approach to recruitment, for ethical reasons and participant safety, participants who had cardiovascular, or neuromuscular conditions were excluded. Despite these limitations, understanding muscle atrophy at the initial, stage prior to sarcopenia, via cautious use of the simple OLST test might be useful for designing and planning appropriate interventions such as nutrition and exercise.

The current study in actively living healthy older women provides novel evidence for the potential of using the one-leg standing balance test in the assessment of low skeletal muscle mass and suggests $55 \mathrm{~s}$ (summed score) as the optimal threshold for detection. We suggest more cross sectional and longitudinal studies to evaluate the derived OLST threshold's ability to identify low muscle mass individuals and explore its potential to investigate other possible factors that are associated with low muscle mass in healthy community dwellers. By identifying lower muscle mass using the OLST, earlier personalised targeting of interventions among the elderly may be possible.

\section{Data availability}

The datasets generated during and/or analysed during the current study are available from the corresponding author on reasonable request.

Supplementary Information The online version contains supplementary material available at https://doi.org/10.1007/s40520-021-01818-X.

Author contributions P.K and C.I.M designed research; P.K and L.H performed research; P.K, G.K.S, G.O.M, H.D, A.G.W, M.T and C.I.M helped P.K analysing and interpreting the results; P.K wrote the manuscript; and L.H, G.K.S, G.O.M, H.D, A.G.W, M.T and C.I.M revised the manuscript.

Funding This work was supported by the Erasmus Mundus Joint Doctorate programme (2011-0015) funded by the European Union as a part of MOVE-AGE for Praval Khanal.

\section{Compliance with ethical standards}

Conflict of interest None of the authors has any conflict of interest to disclose.

Ethics approval The study followed the 1964 Declaration of Helsinki guidelines and was approved by the local ethics committee [Manchester Metropolitan University Ethics Committee; Approval number: 09.02.16 (i)].

Consent to participate All participants provided written informed consent.

Consent to publication Not applicable.

Open Access This article is licensed under a Creative Commons Attribution 4.0 International License, which permits use, sharing, adaptation, distribution and reproduction in any medium or format, as long as you give appropriate credit to the original author(s) and the source, provide a link to the Creative Commons licence, and indicate if changes were made. The images or other third party material in this article are included in the article's Creative Commons licence, unless indicated otherwise in a credit line to the material. If material is not included in the article's Creative Commons licence and your intended use is not permitted by statutory regulation or exceeds the permitted use, you will need to obtain permission directly from the copyright holder. To view a copy of this licence, visit http://creativecommons.org/licenses/by/4.0/. 


\section{References}

1. Cruz-Jentoft AJ et al (2010) Sarcopenia: European consensus on definition and diagnosis Report of the European Working Group on Sarcopenia in Older PeopleA. Age Ageing 39:412-423

2. Janssen I, Heymsfield SB, Ross R (2002) Low relative skeletal muscle mass (sarcopenia) in older persons is associated with functional impairment and physical disability. J Am Geriatr Soc 50:889-896

3. Cheng Y-H et al (2020) Effects of protein supplementation on aerobic training-induced gains in cardiopulmonary fitness, muscle mass, and functional performance in chronic stroke: a randomized controlled pilot study. Clin Nutr 39:2743-2750

4. Cruz-Jentoft AJ et al (2019) Sarcopenia: revised European consensus on definition and diagnosis. Age Ageing 48:16-31

5. Pollock AS et al (2000) What is balance? Clin Rehabil 14:402-406

6. Granacher U, MuehlbauerGruber TM (2012) A qualitative review of balance and strength performance in healthy older adults: impact for testing and training. J Aging Res 2012:16. https://doi. org/10.1155/2012/708905

7. Angelaki DE, Klier EM, Snyder LH (2009) A vestibular sensation: probabilistic approaches to spatial perception. Neuron 64:448-461

8. Sturnieks DL, St George R, Lord SR (2008) Balance disorders in the elderly. Clin Neurophys 38:467-478

9. Jonsson E, Seiger A, Hirschfeld H (2004) One-leg stance in healthy young and elderly adults: a measure of postural steadiness? Clin Biomech 19:688-694

10. Michikawa T et al (2009) One-leg standing test for elderly populations. J Orthop Sci 14:675-685

11. Serra-Prat M, Palomera E (2019) Muscle strength, Sarcopenia and Frailty Associations with balance and gait parameters: a crosssectional Study. Eur J Geriatr Gerontol 1:61-66

12. Bijlsma AY et al (2013) Muscle strength rather than muscle mass is associated with standing balance in elderly outpatients. J Am Med Dir Assoc 14:493-498

13. Vellas BJ et al (1997) One-leg balance is an important predictor of injurious falls in older persons. J Am Geriatr Soc 45:735-738

14. Drusini A et al (2002) One-leg standing balance and functional status in an elderly community-dwelling population in northeast Italy. Aging Clin Exp Res 14:42-46

15. Gillette-Guyonnet $\mathrm{S}$ et al (2000) Body composition and osteoporosis in elderly women. Gerontology 46:189-193

16. Ambrose AF, Paul G, Hausdorff JM (2013) Risk factors for falls among older adults: a review of the literature. Maturitas 75:51-61

17. Cawthon PM et al (2019) Strong relation between muscle mass determined by D3-creatine dilution, physical performance, and incidence of falls and mobility limitations in a prospective cohort of older men. J Gerontol 74:844-852

18. Hawk C et al (2006) Assessment of balance and risk for falls in a sample of community-dwelling adults aged 65 and older. Chiropr Osteopat 14:3

19. Kuh D et al (2005) Grip strength, postural control, and functional leg power in a representative cohort of British men and women: associations with physical activity, health status, and socioeconomic conditions. J Gerontol A Biol Sci Med Sci 60:224-231

20. Netz Y, Argov E (1997) Assessment of functional fitness among independent-older adults: a preliminary report. Percept Mot Skills 84:1059-1074

21. Bohannon RW et al (1984) Decrease in timed balance test scores with aging. Phys Ther 64:1067-1070

22. Wolinsky FD et al (2005) Reproducibility of physical performance and physiologic assessments. J Aging Health 17:111-124

23. Lin MR et al (2004) Psychometric comparisons of the timed up and go, one-leg stand, functional reach, and Tinetti balance measures in community-dwelling older people. J Am Geriatr Soc 52:1343-1348

24. Khanal P et al (2020) Prevalence and association of single nucleotide polymorphisms with sarcopenia in older women depends on definition. Sci Rep 10:1-9

25. Janssen I et al (2000) Estimation of skeletal muscle mass by bioelectrical impedance analysis. J Appl Physiol 89:465-471

26. Faria SL et al (2014) Validation study of multi-frequency bioelectrical impedance with dual-energy X-ray absorptiometry among obese patients. Obes Surg 24:1476-1480

27. He L et al (2020) Associations of combined genetic and epigenetic scores with muscle size and muscle strength: a pilot study in older women. J Cachexia Sarcopenia Muscle 11:1548-1561. https://doi. org/10.1002/jcsm. 12585

28. Khanal $\mathrm{P}$ et al (2020) The association of multiple gene variants with ageing skeletal muscle phenotypes in elderly women. Genes 11:1459

29. Hulens $M$ et al (2001) Study of differences in peripheral muscle strength of lean versus obese women: an allometric approach. Int J Obes 25:676-681

30. Washburn RA, Ficker JL (1999) Physical Activity Scale for the Elderly (PASE): the relationship with activity measured by a portable accelerometer. J Sports Med Phys Fitness 39:336

31. Minetto MA et al (2016) Ultrasound-based detection of low muscle mass for diagnosis of sarcopenia in older adults. PM $\mathrm{R}$ $8: 453-462$

32. Confortin SC et al (2017) Anthropometric indicators as a screening tool for sarcopenia in older adults from Florianópolis, Santa Catarina: EpiFloripa Ageing study. Revista de Nutrição 30:287-296

33. Kim S-E et al (2016) Relative appendicular skeletal muscle mass is associated with isokinetic muscle strength and balance in healthy collegiate men. J Sports Sci 34:2114-2120

34. Auyeung TW et al (2014) Age-associated decline of muscle mass, grip strength and gait speed: a 4-year longitudinal study of 3018 community-dwelling older Chinese. Geriatr Gerontol Int 14:76-84

35. Yang S, Berdine G (2017) The receiver operating characteristic (ROC) curve. Southwest Respir Crit Care Chronic 5:34-36

36. Kolic J et al (2020) Understanding the impact of age, gender, height and body mass index on children's balance. Acta Paediatr 109:175-182

37. Trombetti A et al (2016) Age-associated declines in muscle mass, strength, power, and physical performance: impact on fear of falling and quality of life. Osteoporos Int 27:463-471

38. Hardy R et al (2013) Body mass index, muscle strength and physical performance in older adults from eight cohort studies: the HALCyon programme. PloS One 8:e56483. https://doi.org/10. 1371/journal.pone.0056483

39. Seichi A et al (2014) Determination of the optimal cutoff time to use when screening elderly people for locomotive syndrome using the one-leg standing test (with eyes open). J Orthop Sci 19:620-626

40. Silva TR, Spritzer PM (2017) Skeletal muscle mass is associated with higher dietary protein intake and lower body fat in postmenopausal women: a cross-sectional study. Menopause 24:502-509

41. Mitchell WK et al (2012) Sarcopenia, dynapenia, and the impact of advancing age on human skeletal muscle size and strength; a quantitative review. Front Physiol 3:260

42. Taekema DG et al (2010) Handgrip strength as a predictor of functional, psychological and social health A prospective populationbased study among the oldest old. Age Ageing 39:331-337

43. Lafortuna $\mathrm{C}$ et al (2005) Gender variations of body composition, muscle strength and power output in morbid obesity. Int $\mathrm{J}$ Obes 29:833-841 
44. Vivodtzev I et al (2018) Quadriceps muscle fat infiltration is associated with cardiometabolic risk in COPD. Clin Physiol Funct Imaging 38:788-797

Publisher's Note Springer Nature remains neutral with regard to jurisdictional claims in published maps and institutional affiliations. 